\title{
Review Article \\ Oxidative Stress and Adipocyte Biology: Focus on the Role of AGEs
}

\author{
Florence Boyer, ${ }^{1}$ Jennifer Baraka Vidot, ${ }^{1}$ Alexis Guerin Dubourg, ${ }^{1}$ Philippe Rondeau, ${ }^{1}$ \\ M. Faadiel Essop, ${ }^{2}$ and Emmanuel Bourdon ${ }^{1}$ \\ ${ }^{1}$ UMR DéTROI, Inserm U1188 Diabète Athérothrombose Thérapies Réunion Océan Indien, Université de La Réunion, \\ Plateforme CYROI, Saint Denis de La Réunion, France \\ ${ }^{2}$ Cardio-Metabolic Research Group (CMRG), Department of Physiological Sciences, Stellenbosch University, \\ Stellenbosch, South Africa \\ Correspondence should be addressed to Emmanuel Bourdon; emmanuel.bourdon@univ-reunion.fr
}

Received 17 December 2014; Accepted 6 March 2015

Academic Editor: Jeannette Vasquez-Vivar

Copyright (C) 2015 Florence Boyer et al. This is an open access article distributed under the Creative Commons Attribution License, which permits unrestricted use, distribution, and reproduction in any medium, provided the original work is properly cited.

\begin{abstract}
Diabetes is a major health problem that is usually associated with obesity, together with hyperglycemia and increased advanced glycation endproducts (AGEs) formation. Elevated AGEs elicit severe downstream consequences via their binding to receptors of AGEs (RAGE). This includes oxidative stress and oxidative modifications of biological compounds together with heightened inflammation. For example, albumin (major circulating protein) undergoes increased glycoxidation with diabetes and may represent an important biomarker for monitoring diabetic pathophysiology. Despite the central role of adipose tissue in many physiologic/pathologic processes, recognition of the effects of greater AGEs formation in this tissue is quite recent within the obesity/diabetes context. This review provides a brief background of AGEs formation and adipose tissue biology and thereafter discusses the impact of AGEs-adipocyte interactions in pathology progression. Novel data are included showing how AGEs (especially glycated albumin) may be involved in hyperglycemia-induced oxidative damage in adipocytes and its potential links to diabetes progression.
\end{abstract}

\section{Introduction}

Diabetes and associated pathologies are major health problems with an existing high and continuously rising prevalence worldwide. For example, more than 55 million individuals are burdened with this condition in Europe with it being projected to further increase to 64 million by 2030 [1]. Diabetes is a multifaceted disorder that is characterized by various metabolic derangements, with hyperglycemia as a major culprit. It is also associated with severe complications; for example, diabetes doubles the risk of developing cardiovascular diseases (CVD) that currently constitute the leading cause of mortality in developed countries [2]. Poor lifestyle choices are strongly connected to diabetes development, with especially suboptimal dietary intake and the lack of exercise linked to obesity onset. The latter usually includes excessive fat accumulation in adipose tissues, with such persons exhibiting relatively high body mass index
(BMI) values of typically greater than $30 \mathrm{~kg} / \mathrm{m}^{2}$. However, obesity per se does not represent an underlying medical condition but rather associated complications such as insulin resistance, type 2 diabetes, and CVD [3]. For example, obese persons with a BMI equal to $30 \mathrm{~kg} / \mathrm{m}^{2}$ display a tenfold increase in risk for developing obesity-related pathologies compared to normal weight persons [4]. Together these studies demonstrate that the tremendous increase in obesity and associated pathologies (such as diabetes) constitute a significant global burden of disease that requires serious intervention strategies to counter its growing threat. In addition, a greater understanding of underlying mechanisms linking obesity to associated pathologies is essential as it may lead to the development of novel therapeutic interventions.

Oxidative stress, and more specifically oxidative damage to proteins, is increasingly thought to play a central, mechanistic role in this context as it is associated with modifications in the activities of biological compounds and cellular 
processes that may be linked to pathological complications. In support, the pathophysiologic perturbations connected with obesity-related diabetes are robustly associated with hyperglycemia-induced oxidative stress $[5,6]$. Here oxidative stress may originate from various sources, with the mitochondrion proposed to play a major role as what was previously shown by our laboratory for the heart [7]. Furthermore, our recent data demonstrate that extra-mitochondrial sources such as NADPH oxidases can also generate reactive oxygen species (ROS) in cardiomyoblasts exposed to simulated hyperglycemic conditions [8]. Such oxidative stress is further fueled by excessive ROS production from glucose autoxidation and also the nonenzymatic, covalent attachment of glucose molecules to circulating proteins that results in the formation of glycated proteins and advanced glycation endproducts (AGEs) [9]. Greater AGE availability can in turn lead to downstream consequences, that is, binding to the receptor for AGE (RAGE) on target cells that induces several intracellular phenomena that likely contribute to the onset of diabetic complications (recent review in [10]). Higher systemic glucose levels can therefore lead to modifications of target proteins with severe downstream effects. For example, enhanced glycation of albumin (major protein in circulation) with diabetes significantly impairs its normal antioxidant function, while at the same time it also acquires additional detrimental properties [11, 12].

Despite the pivotal part that adipocytes play in the onset of several physiological/pathological processes, the role of increased AGEs formation in such tissues is not well understood and it is considered a slowly emerging research niche area [13]. For example, the first studies showing the impact of AGE-modified bovine serum albumin (BSA) on adipocytes were only published in 2003 [14, 15]. This minireview will therefore focus on the impact of AGEs-adipocyte interactions in terms of diabetes pathology progression. The background of AGEs formation and adipose tissue biology will initially be reviewed and thereafter the focus will shift to the link between AGEs and adipocytes. We will also include recent data focusing on glycated albumin and its link to hyperglycemiainduced oxidative damage in adipocytes.

\section{AGEs Formation and Receptors}

Several reaction cascades can result in AGEs formation, with the steps leading to glycation known as the Maillard reaction that was discovered by the famous French chemist Louis Camille Maillard during the early 1900s. This results in the nonenzymatic and nonoxidative covalent attachment of glucose molecules to target proteins, lipids, and nucleic acids [16]. Glycoxidation refers to the radical-mediated oxidation reaction of both free and protein-bound sugars [2]. The Amadori rearrangement of glycated proteins gives rise to advanced glycoxidation endproducts (also termed AGEs) [17]. The high variety in such reactions renders AGEs a heterogeneous group of chemically modified proteins [13]. For example, if lysine residues are particularly prone to glycation, the reaction can also affect arginine and cysteine residues leading to the generation of the major AGEs, that is, $\mathrm{N}^{\varepsilon}$ (carboxymethyl)lysine (CML), the crosslinker pentosidine, and S-(carboxymethyl)cysteine (CEC) [12] (Figure 1).

AGEs formation can result both from exogenous sources (dietary intake) and also due to high glucose availability that can trigger the Maillard reaction. AGEs were first identified in food processing technology and used to improve its quality in terms of taste, texture, and sensorial properties. However, recent studies by the Vlassara laboratory revealed the importance of dietary-related AGEs in the development of metabolic disorders and uncovered a novel paradigm; that is, AGEs can precede the onset of diabetes mellitus [18]. Interestingly, other exogenous AGEs sources have also been identified; for example, it is found in cigarette smoke and as a result smokers exhibit relatively high systemic levels [25].

What about AGEs formation within the in vivo context? Such modifications occur on a much longer time scale compared to exogenously supplied AGEs found in various dietary and other sources. Thus in vivo glycation mainly affects circulating proteins with a relatively long half-life such as albumin, the most abundant plasma protein [12]. It is also well established that enhanced albumin glycation with diabetes is associated with the early occurrence of vascular complications, together with functional protein alterations [12, 26-28]. However, AGEs formation and accumulation in diabetic individuals can result from various biochemical reactions, that is, "many roads leading to Rome" [29]. Here it can result from the reaction with highly reactive glucosederived glycolytic intermediates such as glyoxal, methylglyoxal, or 3-deoxyglucosone that react 7- to 200-fold faster than glucose [19]. Of note, such AGEs are not formed solely from carbohydrate metabolism but can also result from lipid oxidation and degradation [20].

The glycation of plasma proteins leads to several downstream consequences and this is initiated by its binding to cell membrane-specific receptors. The different receptors able to recognize AGEs can be schematically divided into three types depending on the signaling pathways subsequently induced (Figure 2). RAGE represents the most studied receptor and is mainly expressed on vascular, endothelial, and smooth muscle cells and on monocyte/macrophage membranes [10, 30]. The RAGE family also includes the AGE-R complex constituted by AGE-R1 80K-H, AGE-R2 OST-48, AGE-R3 galectin-3, SR-A (macrophage scavenger receptor types I and II), and SR-B (SR-B type I and CD36) [23, 30]. Several physiologic and pathophysiologic roles have been reported for receptors able to bind to AGEs [10]. Most are considered as toxic effects, that is, downstream oxidative stress generation and the initiation of inflammatory cascades such as ROSrelated activation of the proinflammatory transcriptional modulator, $\mathrm{NF} \kappa \mathrm{B}[10]$. Conversely, AGE-R1 and R3 compete with RAGE for AGE binding with resultant opposing effects such as the protective role of AGER against ROS formation, oxidative stress, and increasing AGE degradation and urinary excretion [18]. Scavenger receptors are a family of receptors able to recognize oxidized or acetylated low density lipoproteins at macrophage surfaces. Scavenger receptors A and B (CD36 and SR-B, resp.) are involved in the intracellular 


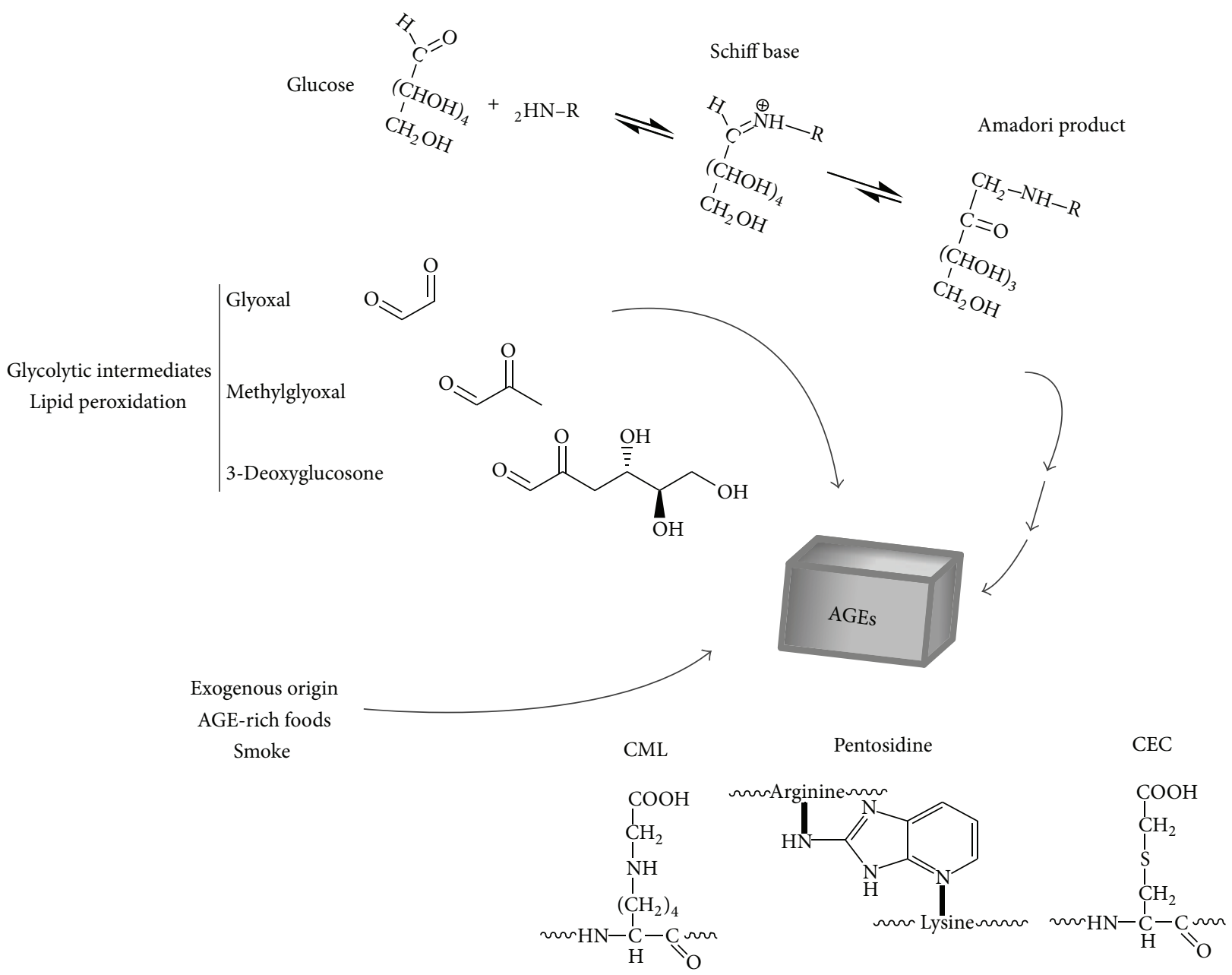

FIGURE 1: Formation of AGEs. AGEs can have different origins such as rearrangements of Amadori products, the latter arising from the glycation reaction. It can also be formed from glycolytic products with an exogenous origin. The glycation reaction mainly affects lysine, arginine, and cysteine residues leading to the formation of the following major AGEs: $\mathrm{N}^{\varepsilon}$-(carboxymethyl)lysine (CML), the crosslinker pentosidine, and S-(carboxymethyl)cysteine (CEC) (adapted from [10, 12, 13, 18-22]).

accumulation of cholesterol and the formation of foam cells from macrophages in the early state of atherosclerosis [31].

As discussed in this section, AGEs have different origins and several receptors play crucial roles in mediating their downstream intracellular effects. In this regard several studies focused on the effects of AGEs on vascular cells and also circulating cells such as monocytes or circulation-derived cells. However, despite the important role of adipocytes in the etiology of diabetes, little is known about the effect of AGEs on such cells. Thus in the following sections an overview of adipose tissue biology and the impact of AGEs on adipocytes will be discussed.

\section{Overview of Adipose Tissue Biology}

Obesity is defined as a condition characterized by excessive fat accumulation and storage [32]. Hence, with caloric abundance, fat is stored as triglycerides (TG) in adipocytes within adipose depots. However, fat stores can also be utilized during times of caloric debt to provide energy substrates by the release of nonesterified fatty acids (NEFA) into circulation [33]. With obesity there is a higher risk for the onset of cardiometabolic diseases and here increased adiposity (especially visceral adiposity) is linked with a greater risk for insulin resistance/type 2 diabetes [34-36]. Although the link(s) between obesity, insulin resistance, and the development of type 2 diabetes is still not fully elucidated [33], several studies show that the dysregulation of adipocyte function is a crucial role player associated with diabetes pathology progression. It is therefore not surprising that numerous investigations are focusing on this problem in order to derive novel therapies that target adipocyte dysregulation within the diabetic milieu [37-39].

The underlying biology of adipose tissue is far more complex than the original concept of its sole function being that of a fat storage depot [40,41]. In addition to TG storage and NEFA release into circulation, adipocytes express and secrete a variety of active biomolecules or "adipokines" that regulate many physiologic processes such as insulin sensitivity, appetite, immunity, and reproduction [42, 43]. 




FIGURE 2: Different types of AGEs receptors. Three major AGEs-receptor pathways are represented: the AGE-R complex constituted by (a) AGE-R1 80K-H, AGE-R2 OST-48, and AGE-R3 galectin-3, (b) RAGE, and (c) CD36 that belongs to SR-B (macrophage scavenger receptor type B) (adapted from $[10,12,23,24]$ ).

Although various processes are implicated in the development of insulin resistance in adipocytes, inflammation and oxidative stress emerge as robust causative factors in this instance $[6,34,44-46]$. In support, a growing number of publications highlight the role of inflammation and oxidative stress and its impact on adipocyte disorders; for example, recent work found increased NF $\kappa \mathrm{B}$-mediated cytokine release from adipocytes isolated from obese individuals [47]. Growth hormone administration also improved glucose intolerance in obese mice presumably by decreasing adipose mass, oxidative stress, and chronic inflammation in visceral fat stores [48]. Moreover, glutathionylated lipid aldehydes, identified as products of adipocyte oxidative stress, result in the activation of macrophage inflammation [49], while an antioxidant molecule attenuated hypoxia-induced oxidative stress, inflammation, and mitochondrial dysfunction in 3T3L1 adipocytes [50].

Among the cytokines originating from adipocytes, secretion of the S100 calcium binding protein B, a RAGE ligand, was recently shown to be enhanced in 3T3 L1 cells incubated under inflammatory conditions and triggered macrophage activation though RAGE [51].
Together these studies demonstrate that oxidative stress and inflammation are crucial pathophysiologic mediators that contribute to adipocyte dysregulation and the onset of various cardiometabolic complications.

Most studies implicating oxidative stress and inflammation in adipocyte pathophysiology employed in vitrobased methodologies with cells exposed to various stressors such as hypoxia, lipopolysaccharides, hydrogen peroxide, and hyperglycemic treatments [52-55]. Thus limited studies have examined the role of AGEs per se in adipocytes, with the detrimental effects of AGEs generally investigated in vascular cells and also circulating cells such as macrophages. The following section will therefore focus on recent, novel data regarding the impact of circulating AGEs on adipocytes.

\section{AGEs Adipocyte Interactions and the Onset of Pathologies}

As discussed earlier, the effects of AGEs on adipocyte function are limited; for example, a PubMed search with the terms "AGEs adipocytes glycation" retrieved only 19 references. What then is known about the effects of AGEs on 



Figure 3: Adipocyte cell line expresses CD36 receptor. SW872 adipocytes were cultured on $12 \mathrm{~mm}$ diameter poly(L-lysine) coverslips until 80\% confluency. Cells were fixed in diluted paraformaldehyde solution ( $4 \% \mathrm{v} / \mathrm{v}$ in PBS) at room temperature for 20 min and stained successively with a primary human CD36 antibody (1:50 in 1\% PBS/BSA) for one hr, followed by incubation with an Alexa Fluor 488 goat anti-rabbit IgG antibody $(1: 1,000)$ for $1 \mathrm{hr}$ and with DAPI solution $(1: 1,000)$ for $10 \mathrm{~min}$ (for staining of the nucleus). Cells were washed 3x in $1 \%$ PBS/BSA between each step of different incubation periods. Cells were imaged using a Nikon eclipse microscope and NIS-Element software (Nikon Corporation, Tokyo, Japan). ${ }^{\# \#} P<0.001$ (vs. HSA) by Student's unpaired $t$ test $(n=3)$.

adipocytes? Studies done thus far have identified the major downstream effects as a heightened inflammatory response as well as the generation of intracellular oxidative stress. For example, a recent study showed that AGEs augmented the expression of the prothrombotic/inflammatory regulator, plasminogen activator inhibitor-1, in rat white adipocytes, by a ROS-dependent pathway [56]. Moreover, glycated BSA increased the adipogenic potential of senescent preadipocytes (in vitro and ex vivo) via the AGEs-RAGE axis together with an impairment of p53 function [57]. Here this occurs by direct binding of RAGE to cytosolic p53 together with the AGEs-RAGE suppression of p53 transcript levels. This in turn enhances the adipogenic potential of preadipocytes, with detrimental long-term effects. Recent experiments performed in our laboratory demonstrated that glycated albumin exposure induced oxidative stress in primary human adipocytes thereby leading to the accumulation of oxidized proteins $[58,59]$. A proteomic-based approach allowed us to also determine preferential protein carbonylation targets in human mature adipocytes treated with glycated versus native albumin [59]. Our studies also revealed greater insights into some of the underlying mechanisms as AGEs-treated adipocytes displayed decreased ubiquitin proteasomal system (UPS) activities and were therefore unable to clear damaged proteins. These data therefore suggest that the origin of accumulated oxidized proteins in AGEs-treated adipocytes likely stems from increased intracellular ROS production together with an impaired UPS $[59,60]$.

What about the receptors for AGE found on adipocyte membranes? Kuniyasu et al. (2003) were the first to discover the presence of CD36 on mouse adipocyte (3T3 L1 cell lines) cell membranes and on human adipocytes from primary cultures that were able to bind and facilitate AGEs endocytosis and degradation [15]. Two years later the same group identified a pathological role for CD36 in AGE binding in adipocytes, that is, resulting in decreased in vivo leptin expression and attenuated insulin sensitivity $[61,62]$. In addition, adipocytes exposed to AGEs exhibited 




(a)



(b)

FIGURE 4: Glycated albumin induces CD36 expression in SW872 adipocytes. (a) SW872 adipocytes were incubated for 24 hr in the absence (PBS) or the presence of $50 \mu \mathrm{M}$ native human serum albumin (HSA) or AGEs constituted by methylglyoxal- (MGO) modified HSA. The relative quantification (\% of fluorescent cells versus control PBS) of CD36 receptors was determined by employing a Becton Dickinson FACScan (BD Biosciences, San Jose, CA) after staining with a primary human CD36 antibody (1:50) for 1 hr, followed by incubation with the secondary Alexa Fluor 488 conjugated anti-rabbit (1:100) and PE-conjugated anti-mouse antibodies (1:100) for an additional hour. Cells were washed with a $1 \%$ PBS/BSA blocking buffer between each incubation step. (b) For the CD36 Western blot, $20 \mu \mathrm{g}$ proteins were isolated from SW872 cell lysates (with different treatments as indicated), separated by SDS-PAGE, and transferred onto a nitrocellulose membrane (Bio-Rad Laboratories, Hercules, CA) using a liquid transfer system. Membranes were soaked overnight with blocking buffer (PBS/0.1\% Tween/1\% BSA) and subsequently incubated in blocking buffer with a primary human CD36 antibody (1:200) for $2 \mathrm{hr}$, followed by incubation with a secondary HRP-conjugated sheep anti-mouse IgG antibody $(1: 2,000)$. Membranes were washed with a blocking buffer $(0.1 \%$ PBS/1\% Tween) between different incubation steps. Protein bands were detected by standard ECL methods (Amersham Biosciences, Amersham, UK) and visualized with a Kodak 2000R Image station (Eastman Kodak, Rochester, NY), and routine densitometric analysis was performed for quantification.

diminished adiponectin expression (usually associated with insulin resistance) [63]. AGEs exposure also caused impaired functionality of adiponectin and this may further contribute to the development of insulin resistance $[64,65]$.

The SW872 cell line has only recently been employed as an adipocyte cell model $[59,66-68]$ and has the advantages of a human origin and also does not require any incubation cocktails to differentiate it into mature adipocytes [66]. We therefore initiated studies using this cell line and found elevated oxidative stress and protein damage in AGEs-treated SW872 adipocytes. Such damaging effects could be blunted with the co-treatment of nutritional antioxidants thereby further implicating oxidative stress in this process $[60,69$, 70]. Unpublished findings from our group also demonstrate, for the first time as far as we are aware, that CD36 is expressed in the SW872 cell line (Figure 3). Furthermore, fluorescenceactivated cell sorting (FACS) and Western blot data show increased CD36 expression in SW872 adipocytes exposed to glycated albumin purified from diabetic persons (Figure 4). However, as there are no data regarding CD36 expression in adipocytes within the diabetic context, further research is needed. This is currently being pursued by our laboratory in order to assess the correlation between AGEs levels and
CD36 expression in adipose tissues isolated from transgenic diabetic mice ( $\mathrm{Db} / \mathrm{Db}$ strain).

\section{Conclusions}

Enhanced AGEs formation and the subsequent tissue and cellular oxidative damage, together with inflammation, are now well established in pathophysiologic disorder progression. Increasing evidence shows that AGEs accumulation in adipose tissue may contribute to obesity-associated insulin resistance. However, the precise nature and mechanisms of AGEs impact on the adipocyte's function are only now slowly emerging and remain poorly understood. The picture is also more complex than what was covered in this minireview, focusing only on the role of circulating AGEs versus intracellular AGEs in adipocytes. Moreover, the human body's defense mechanisms to counter the overproduction of AGEs were not discussed in this paper. This field is a "hot topic" and here dysregulation of the glyoxylase system, constituted by glyoxylase-1 (GLO1) and glyoxylase II (GLO2), is emerging as important contributors to higher AGEs levels [13]. For example, a recent study established that GLO1 overexpression attenuated AGEs and diminished oxidative stress [71]. Thus 
further research is required to gain greater insights into the precise nature of the AGEs-RAGE axis in adipocytes and its relevance within the diabetic milieu, with the focus also on extra- and intracellular AGEs availability and the glyoxylase defense system. Such novel insights may in turn unlock novel pharmaceutical and/or nutritional strategic developments that should help blunt obesity-related insulin resistance progression.

\section{List of Abbreviations}

AGE: Advanced glycation endproduct

BMI: Body mass index

CEC: Carboxymethyl-cysteine

CML: Carboxymethyl-lysine

CVD: Cardiovascular diseases

FACS: Fluorescence-activated cell sorting

GLO: Glyoxylase

NEFA: Nonesterified fatty acids

RAGE: Receptor for AGE

ROS: Reactive oxygen species

TG: Triglycerides.

\section{Conflict of Interests}

The authors declare that there is no conflict of interests regarding the publication of this paper.

\section{Acknowledgments}

This work was supported by the Ministère de l'Enseignement Supérieur et de la Recherche et de l'Outre Mer, the Université de La Réunion, and by the Conseil Régional de La Réunion, France and Europe («Redox project»). Florence Boyer and Jennifer Baraka Vidot are recipients of a fellowship from the Conseil Régional de La Réunion, France and Europe.

\section{References}

[1] D. R. Whiting, L. Guariguata, C. Weil, and J. Shaw, "IDF diabetes atlas: global estimates of the prevalence of diabetes for 2011 and 2030," Diabetes Research and Clinical Practice, vol. 94, no. 3, pp. 311-321, 2011.

[2] S. E. Shoelson, J. Lee, and A. B. Goldfine, "Inflammation and insulin resistance," The Journal of Clinical Investigation, vol. 116, no. 7, pp. 1793-1801, 2006.

[3] P. Trayhurn, "Hypoxia and adipose tissue function and dysfunction in obesity," Physiological Reviews, vol. 93, no. 1, pp. 1-21, 2013.

[4] P. G. Kopelman, "Obesity as a medical problem," Nature, vol. 404, no. 6778, pp. 635-643, 2000.

[5] J.-Y. Youn, K. L. Siu, H. E. Lob, H. Itani, D. G. Harrison, and H. Cai, "Role of vascular oxidative stress in obesity and metabolic syndrome," Diabetes, vol. 63, no. 7, pp. 2344-2355, 2014.

[6] S. Furukawa, T. Fujita, M. Shimabukuro et al., "Increased oxidative stress in obesity and its impact on metabolic syndrome," The Journal of Clinical Investigation, vol. 114, no. 12, pp. 1752-1761, 2004.
[7] R. F. Mapanga, D. Joseph, B. Symington et al., "Detrimental effects of acute hyperglycaemia on the rat heart," Acta Physiologica (Oxf), vol. 210, no. 3, pp. 546-564, 2014.

[8] D. Joseph, C. Kimar, B. Symington, R. Milne, and M. F. Essop, "The detrimental effects of acute hyperglycemia on myocardial glucose uptake," Life Sciences, vol. 105, no. 1-2, pp. 31-42, 2014.

[9] F. Giacco and M. Brownlee, "Oxidative stress and diabetic complications," Circulation Research, vol. 107, no. 9, pp. 10581070, 2010.

[10] C. Ott, K. Jacobs, E. Haucke, A. Navarrete Santos, T. Grune, and A. Simm, "Role of advanced glycation end products in cellular signaling," Redox Biology, vol. 2, no. 1, pp. 411-429, 2014.

[11] M. Roche, P. Rondeau, N. R. Singh, E. Tarnus, and E. Bourdon, "The antioxidant properties of serum albumin," FEBS Letters, vol. 582, no. 13, pp. 1783-1787, 2008.

[12] P. Rondeau and E. Bourdon, "The glycation of albumin: structural and functional impacts," Biochimie, vol. 93, no. 4, pp. 645658, 2011.

[13] K. H. Gaens, C. D. Stehouwer, and C. G. Schalkwijk, "Advanced glycation endproducts and its receptor for advanced glycation endproducts in obesity," Current Opinion in Lipidology, vol. 24, no. 1, pp. 4-11, 2013.

[14] S. Horiuchi, Y. Sakamoto, and M. Sakai, "Scavenger receptors for oxidized and glycated proteins," Amino Acids, vol. 25, no. 34, pp. 283-292, 2003.

[15] A. Kuniyasu, N. Ohgami, S. Hayashi, A. Miyazaki, S. Horiuchi, and H. Nakayama, "CD36-mediated endocytic uptake of advanced glycation end products (AGE) in mouse 3T3-L1 and human subcutaneous adipocytes," FEBS Letters, vol. 537, no. 1-3, pp. 85-90, 2003.

[16] M. P. Cohen, "Clinical, pathophysiological and structure/ function consequences of modification of albumin by Amadoriglucose adducts," Biochimica et Biophysica Acta-General Subjects, vol. 1830, no. 12, pp. 5480-5485, 2013.

[17] S. Pennathur and J. W. Heinecke, "Mechanisms for oxidative stress in diabetic cardiovascular disease," Antioxidants and Redox Signaling, vol. 9, no. 7, pp. 955-969, 2007.

[18] H. Vlassara and G. E. Striker, "AGE restriction in diabetes mellitus: a paradigm shift," Nature Reviews Endocrinology, vol. 7, no. 9, pp. 526-539, 2011.

[19] N. B. Ruderman, J. R. Williamson, and M. Brownlee, "Glucose and diabetic vascular disease," The FASEB Journal, vol. 6, no. 11, pp. 2905-2914, 1992.

[20] A. Stirban, T. Gawlowski, and M. Roden, "Vascular effects of advanced glycation endproducts: clinical effects and molecular mechanisms," Molecular Metabolism, vol. 3, no. 2, pp. 94-108, 2014.

[21] S. Radjei, B. Friguet, C. Nizard, and I. Petropoulos, "Prevention of dicarbonyl-mediated advanced glycation by glyoxalases: implication in skin aging," Biochemical Society Transactions, vol. 42, no. 2, pp. 518-522, 2014.

[22] P. J. Thornalley, A. Langborg, and H. S. Minhas, "Formation of glyoxal, methylglyoxal and 3-deoxyglucosone in the glycation of proteins by glucose," Biochemical Journal, vol. 344, part 1, pp. 109-116, 1999.

[23] N. Ohgami, R. Nagai, M. Ikemoto et al., "CD36, a member of class B scavenger receptor family, is a receptor for advanced glycation end products," Annals of the New York Academy of Sciences, vol. 947, pp. 350-355, 2001. 
[24] P. J. Thornalley, "Cell activation by glycated proteins. AGE receptors, receptor recognition factors and functional classification of AGEs," Cellular and Molecular Biology, vol. 44, no. 7, pp. 1013-1023, 1998.

[25] C. Cerami, H. Founds, I. Nicholl et al., "Tobacco smoke is a source of toxic reactive glycation products," Proceedings of the National Academy of Sciences of the United States of America, vol. 94, no. 25, pp. 13915-13920, 1997.

[26] A. Guerin-Dubourg, A. Catan, E. Bourdon, and P. Rondeau, "Structural modifications of human albumin in diabetes," Diabetes and Metabolism, vol. 38, no. 2, pp. 171-178, 2012.

[27] J. Baraka-Vidot, A. Guerin-Dubourg, F. Dubois, B. Payet, E. Bourdon, and P. Rondeau, "New insights into deleterious impacts of in vivo glycation on albumin antioxidant activities," Biochimica et Biophysica Acta, vol. 1830, no. 6, pp. 3532-3541, 2013.

[28] J. Baraka-Vidot, A. Guerin-Dubourg, E. Bourdon, and P. Rondeau, "Impaired drug-binding capacities of in vitro and in vivo glycated albumin," Biochimie, vol. 94, no. 9, pp. 1960-1967, 2012.

[29] N. C. Chilelli, S. Burlina, and A. Lapolla, "AGEs, rather than hyperglycemia, are responsible for microvascular complications in diabetes: a 'glycoxidation-centric' point of view," Nutrition, Metabolism \& Cardiovascular Diseases, vol. 23, no. 10, pp. 913919, 2013.

[30] Y. Miki, H. Dambara, Y. Tachibana, K. Hirano, M. Konishi, and M. Beppu, "Macrophage recognition of toxic advanced glycosylation end products through the macrophage surfacereceptor nucleolin," Biological and Pharmaceutical Bulletin, vol. 37, no. 4, pp. 588-596, 2014.

[31] M. Freeman, Y. Ekkel, L. Rohrer et al., "Expression of type I and type II bovine scavenger receptors in Chinese hamster ovary cells: lipid droplet accumulation and nonreciprocal cross competition by acetylated and oxidized low density lipoprotein," Proceedings of the National Academy of Sciences of the United States of America, vol. 88, no. 11, pp. 4931-4935, 1991.

[32] “obesity," in Merriam Webster Dictionnary, 2014, http://www .merriam-webster.com/dictionary/obesity.

[33] M. Lafontan, "Fat cells: afferent and efferent messages define new approaches to treat obesity," Annual Review of Pharmacology and Toxicology, vol. 45, pp. 119-146, 2005.

[34] G. S. Hotamisligil, "Inflammation and metabolic disorders," Nature, vol. 444, no. 7121, pp. 860-867, 2006.

[35] A. P. Rocchini, "Childhood obesity and a diabetes epidemic," The New England Journal of Medicine, vol. 346, no. 11, pp. 854855, 2002.

[36] B. Balkau, J. E. Deanfield, J.-P. Després et al., "International day for the evaluation of abdominal obesity (IDEA): A study of waist circumference, cardiovascular disease, and diabetes mellitus in 168000 primary care patients in 63 countries," Circulation, vol. 116, no. 17, pp. 1942-1951, 2007.

[37] A. B. Mayerson, R. S. Hundal, S. Dufour et al., "The effects of rosiglitazone on insulin sensitivity, lipolysis, and hepatic and skeletal muscle triglyceride content in patients with type 2 diabetes," Diabetes, vol. 51, no. 3, pp. 797-802, 2002.

[38] R. Rahimi, S. Nikfar, B. Larijani, and M. Abdollahi, "A review on the role of antioxidants in the management of diabetes and its complications," Biomedicine and Pharmacotherapy, vol. 59, no. 7, pp. 365-373, 2005.

[39] P. Arner, "The adipocyte in insulin resistance: key molecules and the impact of the thiazolidinediones," Trends in Endocrinology and Metabolism, vol. 14, no. 3, pp. 137-145, 2003.
[40] D. Thompson, F. Karpe, M. Lafontan, and K. Frayn, "Physical activity and exercise in the regulation of human adipose tissue physiology," Physiological Reviews, vol. 92, no. 1, pp. 157-191, 2012.

[41] E. Oliveros, V. K. Somers, O. Sochor, K. Goel, and F. LopezJimenez, "The concept of normal weight obesity," Progress in Cardiovascular Diseases, vol. 56, no. 4, pp. 426-433, 2014.

[42] M. Lafontan, "Historical perspectives in fat cell biology: the fat cell as a model for the investigation of hormonal and metabolic pathways," The American Journal of Physiology-Cell Physiology, vol. 302, no. 2, pp. C327-C359, 2012.

[43] M. Lafontan, "Adipose tissue and adipocyte dysregulation," Diabetes and Metabolism, vol. 40, no. 1, pp. 16-28, 2014.

[44] K. E. Wellen and G. S. Hotamisligil, "Inflammation, stress, and diabetes," The Journal of Clinical Investigation, vol. 115, no. 5, pp. 1111-1119, 2005.

[45] N. Hosogai, A. Fukuhara, K. Oshima et al., "Adipose tissue hypoxia in obesity and its impact on adipocytokine dysregulation," Diabetes, vol. 56, no. 4, pp. 901-911, 2007.

[46] M. F. Gregor and G. S. Hotamisligil, “Thematic review series: adipocyte biology. Adipocyte stress: the endoplasmic reticulum and metabolic disease," Journal of Lipid Research, vol. 48, no. 9, pp. 1905-1914, 2007.

[47] F. Tourniaire, B. Romier-Crouzet, J. H. Lee et al., "Chemokine expression in inflamed adipose tissue is mainly mediated by NF$\kappa$ B," PLoS ONE, vol. 8, no. 6, Article ID e66515, 2013.

[48] M. Fukushima, Y. Okamoto, H. Katsumata et al., "Growth hormone ameliorates adipose dysfunction during oxidative stress and inflammation and improves glucose tolerance in obese mice," Hormone and Metabolic Research, vol. 46, no. 09, pp. 656-662, 2014.

[49] B. I. Frohnert, E. K. Long, W. S. Hahn, and D. A. Bernlohr, "Glutathionylated lipid aldehydes are products of adipocyte oxidative stress and activators of macrophage inflammation," Diabetes, vol. 63, no. 1, pp. 89-100, 2014.

[50] A. Priyanka, V. M. Nisha, S. S. Anusree, and K. G. Raghu, "Bilobalide attenuates hypoxia induced oxidative stress, inflammation, and mitochondrial dysfunctions in 3T3-L1 adipocytes via its antioxidant potential," Free Radical Research, vol. 48, no. 10, pp. 1206-1217, 2014.

[51] A. Fujiya, H. Nagasaki, Y. Seino et al., "The role of S100B in the interaction between adipocytes and macrophages," Obesity, vol. 22, no. 2, pp. 371-379, 2014.

[52] A. Priyanka, S. S. Anusree, V. M. Nisha, and K. G. Raghu, "Curcumin improves hypoxia induced dysfunctions in 3T3-L1 adipocytes by protecting mitochondria and down regulating inflammation," BioFactors, vol. 40, no. 5, pp. 513-523, 2014.

[53] P. Baret, A. Septembre-Malaterre, M. Rigoulet et al., "Dietary polyphenols preconditioning protects 3T3-L1 preadipocytes from mitochondrial alterations induced by oxidative stress," International Journal of Biochemistry and Cell Biology, vol. 45, no. 1, pp. 167-174, 2013.

[54] F. S. Lira, J. C. Rosa, G. D. Pimentel et al., "Both adiponectin and interleukin-10 inhibit LPS-induced activation of the NF- $\kappa \mathrm{B}$ pathway in 3T3-L1 adipocytes," Cytokine, vol. 57, no. 1, pp. 98$106,2012$.

[55] Y. Lin, A. H. Berg, P. Iyengar et al., "The hyperglycemiainduced inflammatory response in adipocytes: the role of reactive oxygen species," The Journal of Biological Chemistry, vol. 280, no. 6, pp. 4617-4626, 2005. 
[56] Y. Uchida, K.-I. Ohba, T. Yoshioka, K. Irie, T. Muraki, and Y. Maru, "Cellular carbonyl stress enhances the expression of plasminogen activator inhibitor-1 in rat white adipocytes via reactive oxygen species-dependent pathway," Journal of Biological Chemistry, vol. 279, no. 6, pp. 4075-4083, 2004.

[57] C.-Y. Chen, A. M. Abell, Y. S. Moon, and K.-H. Kim, "An advanced glycation end product (AGE)-receptor for AGEs (RAGE) axis restores adipogenic potential of senescent preadipocytes through modulation of p53 protein function," Journal of Biological Chemistry, vol. 287, no. 53, pp. 4449844507, 2012.

[58] S. Chesne, P. Rondeau, S. Armenta, and E. Bourdon, "Effects of oxidative modifications induced by the glycation of bovine serum albumin on its structure and on cultured adipose cells," Biochimie, vol. 88, no. 10, pp. 1467-1477, 2006.

[59] N. R. Singh, P. Rondeau, L. Hoareau, and E. Bourdon, "Identification of preferential protein targets for carbonylation in human mature adipocytes treated with native or glycated albumin," Free Radical Research, vol. 41, no. 10, pp. 1078-1088, 2007.

[60] N. R. Singh, P. Rondeau, and E. Bourdon, "Identification of upregulated low molecular weight proteins in human adipocytes treated with glycoxidized albumin," The Open Obesity Journal, vol. 2, no. 1, pp. 110-115, 2009.

[61] Y. Unno, M. Sakai, Y.-I. Sakamoto et al., "Glycolaldehydemodified bovine serum albumin downregulates leptin expression in mouse adipocytes via a CD36-mediated pathway," Annals of the New York Academy of Sciences, vol. 1043, pp. 696701, 2005.

[62] S. Horiuchi, Y. Unno, H. Usui et al., "Pathological roles of advanced glycation end product receptors SR-A and CD36," Annals of the New York Academy of Sciences, vol. 1043, pp. 671675, 2005.

[63] S. Maeda, T. Matsui, M. Takeuchi, and S.-I. Yamagishi, "Pigment epithelium-derived factor (PEDF) blocks advanced glycation end products (AGEs)-RAGE-induced suppression of adiponectin mRNA level in adipocytes by inhibiting NADPH oxidase-mediated oxidative stress generation," International Journal of Cardiology, vol. 152, no. 3, pp. 408-410, 2011.

[64] N. Frizzell, M. Lima, and J. W. Baynes, "Succination of proteins in diabetes," Free Radical Research, vol. 45, no. 1, pp. 101-109, 2011.

[65] N. Frizzell, M. Rajesh, M. J. Jepson et al., "Succination of thiol groups in adipose tissue proteins in diabetes: succination inhibits polymerization and secretion of adiponectin," The Journal of Biological Chemistry, vol. 284, no. 38, pp. 2577225781, 2009.

[66] H. Wassef, L. Bernier, J. Davignon, and J. S. Conn, "Synthesis and secretion of apoC-I and apoE during maturation of human SW872 liposarcoma cells," Journal of Nutrition, vol. 134, no. 11, pp. 2935-2941, 2004.

[67] L. Izem and R. E. Morton, "Possible role for intracellular cholesteryl ester transfer protein in adipocyte lipid metabolism and storage," The Journal of Biological Chemistry, vol. 282, no. 30, pp. 21856-21865, 2007.

[68] G. Vassiliou and R. McPherson, "Role of cholesteryl ester transfer protein in selective uptake of high density lipoprotein cholesteryl esters by adipocytes," Journal of Lipid Research, vol. 45, no. 9, pp. 1683-1693, 2004.

[69] D. Ramful, E. Tarnus, P. Rondeau, C. R. Da Silva, T. Bahorun, and E. Bourdon, "Citrus fruit extracts reduce advanced glycation end products (AGEs)- and $\mathrm{H}_{2} \mathrm{O}_{2}$-induced oxidative stress in human adipocytes," Journal of Agricultural and Food Chemistry, vol. 58, no. 20, pp. 11119-11129, 2010.

[70] M. Roche, E. Tarnus, P. Rondeau, and E. Bourdon, "Effects of nutritional antioxidants on AAPH- or AGEs-induced oxidative stress in human SW872 liposarcoma cells," Cell Biology and Toxicology, vol. 25, no. 6, pp. 635-644, 2009.

[71] O. Brouwers, P. M. Niessen, I. Ferreira et al., "Overexpression of glyoxalase-I reduces hyperglycemia-induced levels of advanced glycation end products and oxidative stress in diabetic rats," Journal of Biological Chemistry, vol. 286, no. 2, pp. 1374-1380, 2011. 


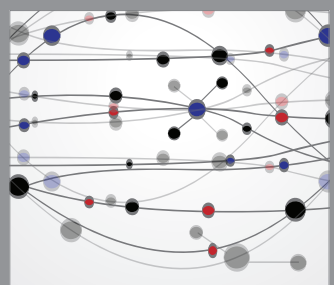

The Scientific World Journal
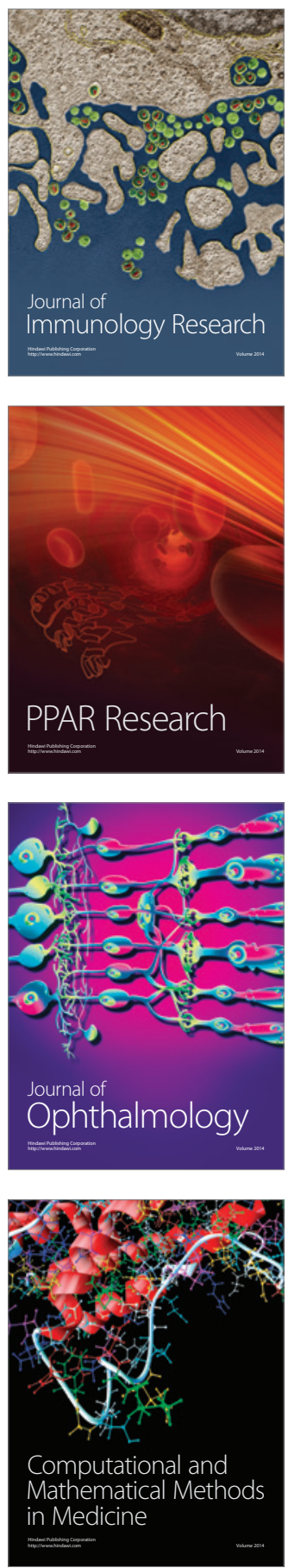

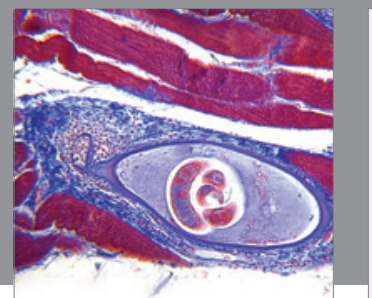

Gastroenterology

Research and Practice
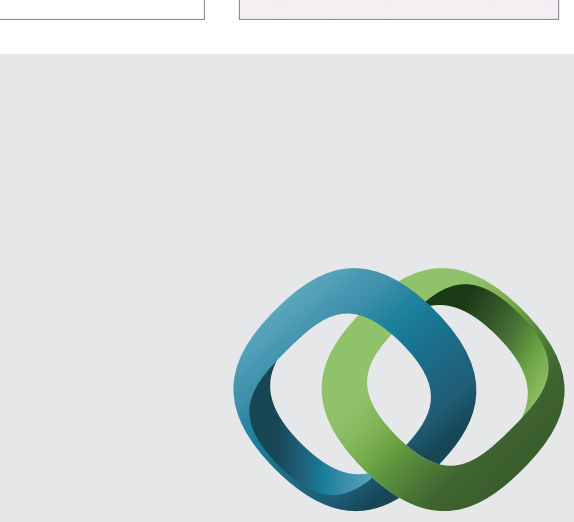

\section{Hindawi}

Submit your manuscripts at

http://www.hindawi.com

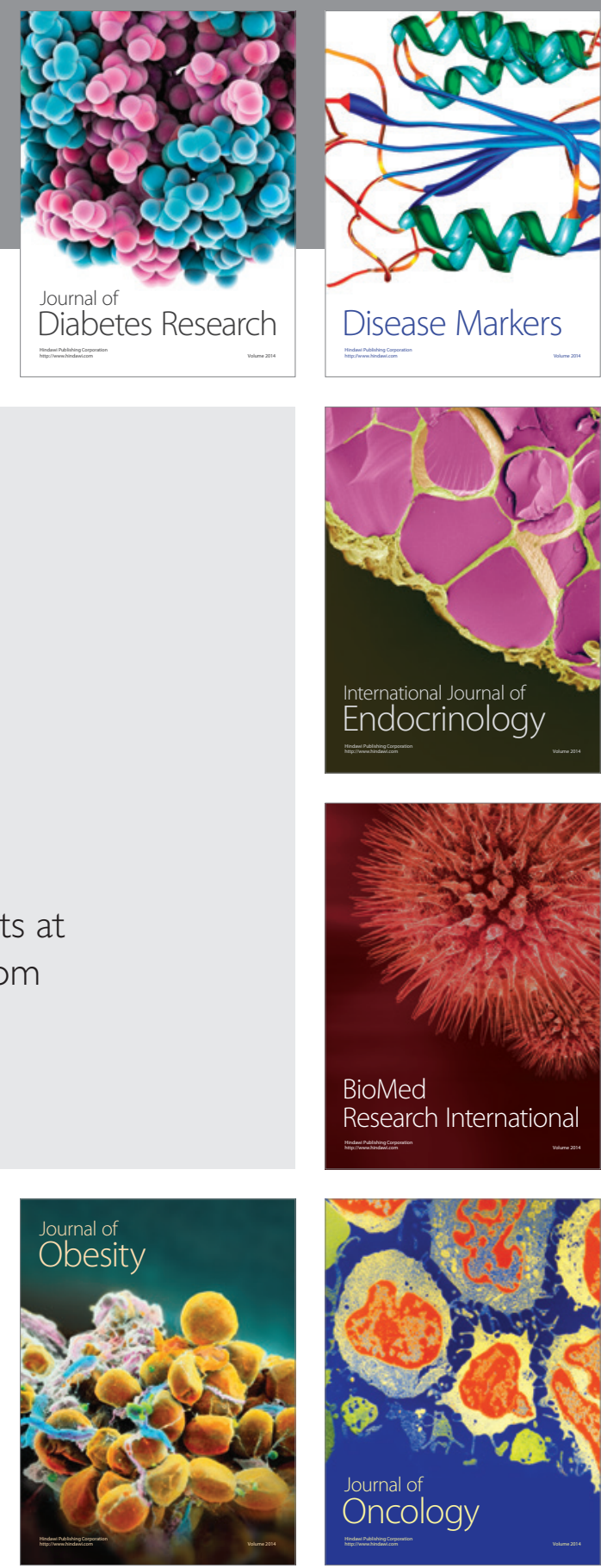

Disease Markers
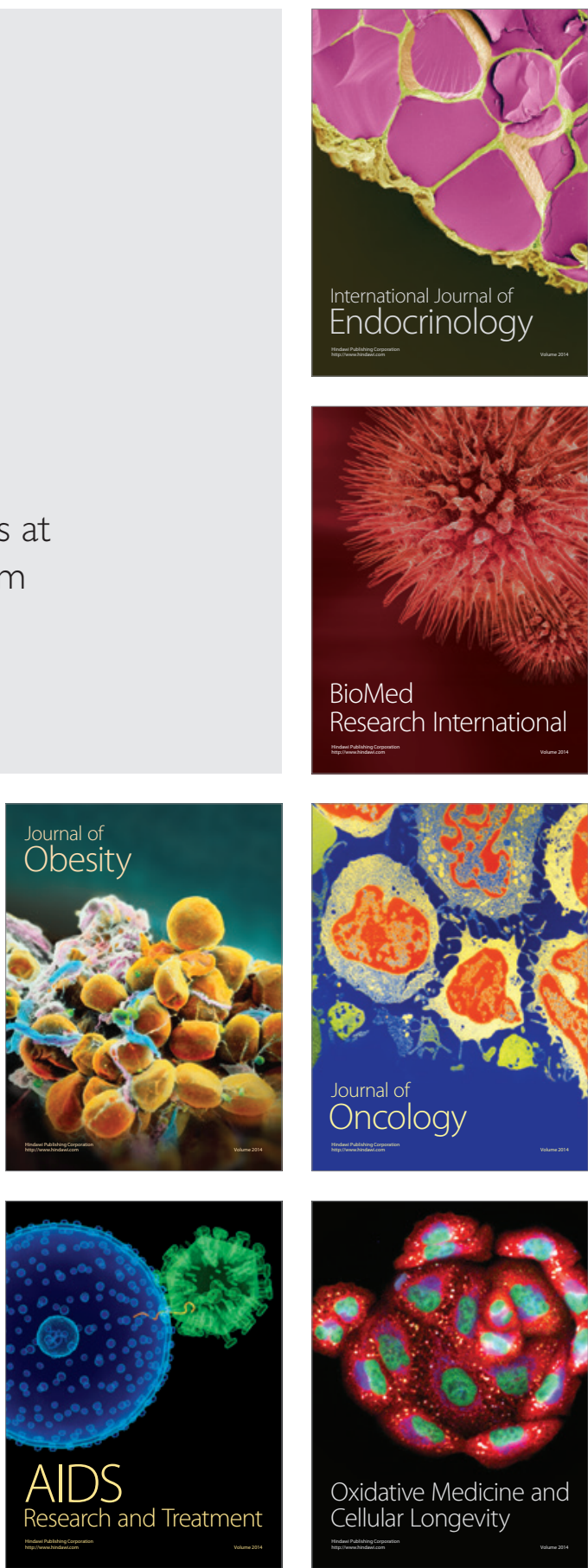